\title{
Membership Functions Representing a Number vs. Representing a Set: Proof of Unique Reconstruction
}

\author{
Hung T. Nguyen \\ New Mexico State University - Main Campus, hunguyen@nmsu.edu \\ Vladik Kreinovich \\ The University of Texas at El Paso, vladik@utep.edu \\ Olga Kosheleva \\ The University of Texas at El Paso, olgak@utep.edu
}

Follow this and additional works at: https://scholarworks.utep.edu/cs_techrep

Part of the Computer Sciences Commons, and the Mathematics Commons

Comments:

Technical Report: UTEP-CS-15-96a

To appear in Proceedings of the 2016 IEEE International Conference on Fuzzy Systems FUZZIEEE'2016, Vancouver, Canada, July 24-29, 2016.

\section{Recommended Citation}

Nguyen, Hung T.; Kreinovich, Vladik; and Kosheleva, Olga, "Membership Functions Representing a Number vs. Representing a Set: Proof of Unique Reconstruction" (2016). Departmental Technical Reports (CS). 982.

https://scholarworks.utep.edu/cs_techrep/982

This Article is brought to you for free and open access by the Computer Science at ScholarWorks@UTEP. It has been accepted for inclusion in Departmental Technical Reports (CS) by an authorized administrator of ScholarWorks@UTEP. For more information, please contact Iweber@utep.edu. 


\section{Membership Functions Representing a Number vs. Representing a Set: Proof of Unique Reconstruction}

\author{
Hung T. Nguyen \\ Department of Mathematical Sciences \\ New Mexico State University \\ Las Cruces, New Mexico 88008, USA \\ and Faculty of Economics \\ Chiang Mai University, Thailand \\ Email: hunguyen@nmsu.edu
}

\author{
Vladik Kreinovich and Olga Kosheleva \\ University of Texas at El Paso \\ El Paso, Texas 79968, USA \\ Email:vladik@utep.edu,olgak@utep.edu
}

\begin{abstract}
In some cases, a membership function $\mu(x)$ represents an unknown number, but in many other cases, it represents an unknown crisp set. In this case, for each crisp set $S$, we can estimate the degree $\mu(S)$ to which this set $S$ is the desired one. A natural question is: once we know the values $\mu(S)$ corresponding to all possible crisp sets $S$, can we reconstruct the original membership function? In this paper, we show that the original membership function $\mu(x)$ can indeed be uniquely reconstructed from the values $\mu(S)$.
\end{abstract}

\section{Formulation of THE PROBlem}

Representing a number vs. representing a set. Fuzzy sets (see, e.g., [1], [5], [6]) are used in two different situations.

In some cases, a fuzzy set is used to represent a number. For example, we ask a person how old is Mary, and this person replies that Mary is young. In this case, there is an actual number representing age. However, we do not know the exact value of this number. Instead, we have a fuzzy set (membership function) that describes our uncertain knowledge about this value.

Each value $\mu(x)$ of this membership function is our degree of confidence that the corresponding value $x$ has the desired property - e.g., that a person of age $x$ is young.

In other cases, a fuzzy set is used to represent not a single crisp value, but rather a whole crisp set. For example, when designing a control system for an autonomous car, we can ask a driver which velocities are safe on a certain road segment. In reality, there is a (crisp) set of such values. However, we do not know this set. Instead, we have a fuzzy set that describes our uncertain knowledge about this unknown set.

A membership function representing a set: analysis of the situation. Let us consider the situation when a membership function $\mu: X \rightarrow[0,1]$ on a universal set $X$ represent an unknown crisp set $U \subseteq X$.

For example, we want to know what are the possible values of a control that guarantee the desired control objective. Based on imprecise (fuzzy) expert information, we conclude that this set corresponds to all the numbers which are approximately equal to 1.5 plus minus 0.2 . This is a fuzzy description of an unknown crisp set.
In this situation, the meaning of the membership function $\mu(x)$ is different: for every element $x \in X$, the value $\mu(x)$ is our degree of confidence that this element $x$ belongs to the (unknown) set $U$. Correspondingly, if we use the usual negation operation $\neg(a)=1-a$, then our degree of confidence that the element $y$ does not belong to the actual set $U$ is equal to $1-\mu(y)$.

Fuzziness means that we do not know the actual set $U$ exactly. In other words, several different crisp sets $S$ are possible candidates for the unknown actual set $U$. For each crisp set $S$, let us estimate our degree of confidence $\mu(S)$ that this set $S$ is the set $U$.

The equality $S=U$ means that:

- for every $x \in S$, we have $x \in U$, and

- for every $y \notin S$, we have $y \notin U$.

In other words, if we consider all the elements $x_{1}, x_{2}, \ldots$ that are contained in the set $S$ and all the elements $y_{1}, \ldots, y_{m}, \ldots$ that are not contained in the crisp set $S$, then the equality $S=U$ means that

$x_{1} \in U$ and $x_{1} \in U$ and $\ldots$ and $y_{1} \notin U$ and $y_{2} \notin U$ and ...

Let us use the standard fuzzy logic methodology to estimate the degree of confidence in this statement.

The above statement is obtained from the elementary statements $x_{i} \in U$ and $y_{j} \in U$ by using propositional connectives "and" and "not". For each elementary statement $x \in U$, by the above interpretation of the membership function, our degree of confidence in this elementary statement is equal to $\mu(x)$. If we use the usual fuzzy negation $\neg(a)=1-a$, then our degree of confidence that $y \notin S$ is equal to $1-\mu(y)$.

According to the usual fuzzy methodology, our degree of confidence in the above "and"-statement (that represents the equality $U=S$ ) can be thus obtained by applying an appropriate "and"-operation (t-norm) to the corresponding degrees of confidence that $x \in U$ and that $y \notin U$.

The main application of fuzzy logic is when the Universe of discourse is either the whole real line, or an interval on the real line. In this case, we have infinitely many possible values $x$. Thus, we will get infinitely many degrees $\mu(x)$ or 
$1-\mu(y)$. It therefore makes sense to require that when we apply the "and"-operation to the resulting degrees, we get a non-trivial value (i.e., we do not always get 0 ).

In fuzzy logic, there are many possible "and"-operations (tnorms); see, e.g., [1], [5]. However, for most of them (e.g., for the algebraic product) the result of applying this operation to infinitely many values is 0 , even when we apply it to several equal degrees. Among the most widely used t-norms, the only "and"-operation for which the result is in this case non- 0 is minimum.

Because of this fact, to get a meaningful value $\mu(S)$, we will use min to combine degrees $\mu(x)$ corresponding to $x \in S$ and degrees $1-\mu(y)$ corresponding to $y \notin S$. As a result, we get the following formula:

$$
\mu(S)=\min \left(\inf _{x \in S} \mu(x), \inf _{y \notin S}(1-\mu(y))\right) .
$$

\section{Comments.}

- Please note that this expression is somewhat similar to the expression for the possibility degree

$$
\operatorname{Poss}(S)=\sup _{x \in S} \mu(x)
$$

and the corresponding degree of belief

$$
\operatorname{Bel}(S)=1-\operatorname{Poss}(-S)=\inf _{x \in S} \mu(x),
$$

where $-S$ denotes a complement to a set $S$. One can easily see, however, that our degree $\rho(S)$ cane described in terms of plausibility and belief, as

$$
\begin{gathered}
\mu(S)=\min (\operatorname{Bel}(S), \operatorname{Bel}(-S))= \\
\min (\operatorname{Bel}(S), 1-\operatorname{Poss}(S)) .
\end{gathered}
$$

- At first glance, it may seem that in this situation, we could also use a probabilistic approach. It is indeed possible to use the probabilistic approach in situations when the universe of discourse consists of finitely many elements. In this case, if we denote the probability that $x \in S$ by $p(x)$, then the probability that $y \notin S$ is equal to

$$
1-p(y)
$$

Thus, if we make a usual probabilistic assumption that events $x \in S$ corresponding to different values $x$ are independent, we get the following formula for the probability

$$
\operatorname{Prob}(S=U)=\left(\prod_{i} p\left(x_{i}\right)\right) \cdot\left(\prod_{j}\left(1-p\left(y_{j}\right)\right)\right)
$$

As we have mentioned earlier, in situations when we have infinitely many values $x_{i}$ and $y_{j}$, this product tends to 0 - i.e., becomes meaningless. Thus, in general, it is not possible to use the probabilistic approach in this situation.

Natural question. We have shown how, if we know the original membership function $\mu(x)$, we can determine the degree $\mu(S)$ for each crisp set $S$. A natural question is: how uniquely can we reconstruct $\mu(x)$ from $\mu(S)$ ?

In other words, if we know the value $\mu(S)$ for every crisp set $S$, can we uniquely reconstruct the original membership function $\mu(x)$ ?

Comment. At first glance, it may seem that this reconstruction is easy: e.g., to find $\mu(a)$, why not take $S=\{a\}$ ? However, one can easily see that this simple approach does not work. For example, if $\mu\left(x_{0}\right)=1$, and we want to find $\mu(a)$ for some $a \neq x_{0}$, then for $x_{0} \notin\{a\}$, we have $1-\mu\left(x_{0}\right)=1-1=0$. Thus, $\inf _{y \notin\{a\}}(1-\mu(y))=0$, and so,

$$
\mu(\{a\})=\min \left(\inf _{x \in\{a\}} \mu(x), \inf _{y \notin\{a\}}(1-\mu(y))\right)=0,
$$

irrespective of what is the actual value of $\mu(a)$.

We therefore need more sophisticated techniques for reconstructing $\mu(x)$ from $\mu(S)$.

\section{MAIN RESULT}

Proposition 1. Let $\mu(x)$ and $\mu^{\prime}(x)$ be membership functions, and let $\mu(S)$ and $\mu^{\prime}(S)$ be corresponding functions (1). Then, if $\mu(S)=\mu^{\prime}(S)$ for all crisp sets $S \subseteq X$, then $\mu(x)=\mu^{\prime}(x)$ for all $x$.

Comment. In other words, the original membership function $\mu(x)$ can indeed be uniquely reconstructed if we know the values $\mu(S)$ for all crisp sets $S$.

Proof. The proof of the main result consists of several lemmas.

Lemma 1. For every $a \in X$,

$$
\mu(a)<0.5 \Leftrightarrow \exists S(\mu(S \cup\{a\})<\mu(S-\{a\})) .
$$

\section{Proof of Lemma 1.}

$1^{\circ}$. Let us first prove that if $\mu(a)<0.5$, then there exists a set $S$ for which $\mu(S \cup\{a\})<\mu(S-\{a\})$.

Indeed, as such a set $S$, we can take $S=\{x: \mu(x) \geq 0.5\}$. In this case, $a \notin S$, so $S-\{a\}=S$ and thus,

$$
\mu(S-\{a\})=\mu(S) .
$$

For the selected set $S$, for all $x \in S$, we have $\mu(x) \geq 0.5$. Thus,

$$
\inf _{x \in S} \mu(x) \geq 0.5 .
$$

For all values $y \notin S$, we have $\mu(y)<0.5$ hence

$$
1-\mu(y)>0.5 \text {. }
$$

Thus, we have

$$
\inf _{y \notin S}(1-\mu(y)) \geq 0.5 \text {. }
$$

Therefore,

$$
\begin{gathered}
\mu(S-\{a\})=\mu(S)= \\
\min \left(\inf _{x \in S} \mu(x), \inf _{y \notin S}(1-\mu(y))\right) \geq 0.5 .
\end{gathered}
$$


On the other hand, for the set $S \cup\{a\}$, we have $\mu(a)<0.5$ for the element $a \in S \cup\{a\}$, thus

$$
\inf _{x \in S \cup\{a\}} \mu(x) \leq \mu(a)<0.5 \text {. }
$$

Therefore,

$$
\begin{gathered}
\mu(S \cup\{a\})=\min \left(\inf _{x \in S \cup\{a\}} \mu(x), \inf _{y \notin S \cup\{a\}}(1-\mu(y))\right) \leq \\
\inf _{x \in S \cup\{a\}} \mu(x)<0.5 .
\end{gathered}
$$

Thus here, $\mu(S \cup\{a\})<0.5 \leq \mu(S-\{a\})$, so indeed

$$
\mu(S \cup\{a\})<\mu(S-\{a\}) .
$$

The existence of such a set $S$ is proven.

$2^{\circ}$. To complete the proof of the lemma, let us now prove that if there exists a set $S$ for which $\mu(S \cup\{a\})<\mu(S-\{a\})$, then $\mu(a)<0.5$.

Indeed, both $\mu(S \cup\{a\})$ and $\mu(S-\{a\})$ are minima of infinitely many terms. Most of these terms are the same, the only difference is the term corresponding to $x=a$ :

- in $\mu(S \cup\{a\})$, we have the term $\mu(a)$ corresponding to $a \in S \cup\{a\}$, while

- in $\mu(S-\{a\})$, we have the term $1-\mu(a)$ corresponding to $a \notin S-\{a\}$.

If we had $\mu(a) \geq 0.5$, then we would have $\mu(a) \geq 1-\mu(a)$, and thus, we would have $\mu(S \cup\{a\}) \geq \mu(S-\{a\})$. So, from the fact that $\mu(S \cup\{a\})<\mu(S-\{a\})$, we conclude that we cannot have $\mu(a) \geq 0.5$, and thus, we must have $\mu(a)<0.5$.

The lemma is proven.

Lemma 2. For every $a \in X$,

$$
\mu(a)>0.5 \Leftrightarrow \exists S(\mu(S \cup\{a\})>\mu(S-\{a\})) .
$$

\section{Proof of Lemma 2.}

$1^{\circ}$. Let us first prove that if $\mu(a)>0.5$, then there exists a set $S$ for which $\mu(S \cup\{a\})>\mu(S-\{a\})$.

Indeed, as such a set $S$, we can take $S=\{x: \mu(x) \geq 0.5\}$. In this case, $a \in S$, so $S \cup\{a\}=S$ and thus,

$$
\mu(S \cup\{a\})=\mu(S) .
$$

As we have shown in the proof of Lemma 1, for this set $S$, we have $\mu(S) \geq 0.5$. Thus,

$$
\mu(S \cup\{a\})=\mu(S) \geq 0.5 .
$$

On the other hand, for the set $S-\{a\}$, we have $1-\mu(a)<0.5$ for $a \notin S-\{a\}$, thus

$$
\inf _{y \notin S-\{a\}}(1-\mu(y)) \leq 1-\mu(a)<0.5 .
$$

Therefore,

$$
\mu(S-\{a\})=\min \left(\inf _{x \in S-\{a\}} \mu(x), \inf _{y \notin S-\{a\}}(1-\mu(y))\right) \leq
$$

$$
\inf _{y \notin S-\{a\}}(1-\mu(y))<0.5 .
$$

Thus here, $\mu(S-\{a\})<0.5 \leq \mu(S \cup\{a\})$, so indeed

$$
\mu(S \cup\{a\})>\mu(S-\{a\}) .
$$

The existence of such a set $S$ is proven.

$2^{\circ}$. To complete the proof of the lemma, let us now prove that if there exists a set $S$ for which $\mu(S \cup\{a\})>\mu(S-\{a\})$, then $\mu(a)>0.5$. Indeed, both $\mu(S \cup\{a\})$ and $\mu(S-\{a\})$ are minima of infinitely many terms. Most of these terms are the same, the only difference is the term corresponding to $x=a$ :

- in $\mu(S \cup\{a\})$, we have the term $\mu(a)$ corresponding to $a \in S \cup\{a\}$, while

- in $\mu(S-\{a\})$, we have the term $1-\mu(a)$ corresponding to $a \notin S-\{a\}$.

If we had $\mu(a) \leq 0.5$, then we would have $\mu(a) \leq 1-\mu(a)$, and thus, we would have $\mu(S \cup\{a\}) \leq \mu(S-\{a\})$. So, from the fact that $\mu(S \cup\{a\})>\mu(S-\{a\})$, we conclude that we cannot have $\mu(a) \leq 0.5$, and thus, we must have $\mu(a)>0.5$.

The lemma is proven.

Discission. According to Lemmas 1 and 2, once we know the values $\mu(S)$ for all crisp sets $S$, we can then, for each element $a \in X$, check whether $\mu(a)<0.5$ and whether $\mu(a)>0.5$.

If for some element $a \in X$, none of these two inequalities is satisfied, then we can conclude that $\mu(a)=0.5$. So, for these elements $a$, we can indeed reconstruct the value $\mu(a)$.

Let us show that we can also reconstruct the value $\mu(a)$ also for the elements $a$ for which $\mu(a)<0.5$ or $\mu(a)>0.5$.

Lemma 3. If $\mu(a)<0.5$, then

$$
\mu(a)=\sup _{S: a \in S} \mu(S) .
$$

Comment 1. Thus, for elements $a$ for which $\mu(a)<0.5$, we can indeed uniquely reconstruct the value $\mu(a)$ from the values $\mu(S)$.

Comment 2. The reconstruction formula is not only mathematically correct (see proof below), it also makes common sense: we say that an element $a$ is possible if there exists a set $S$ containing this element $a$ which is possible. From the common sense viewpoint, "there exists" means "or": either one of the sets $S$ containing $a$ is possible, or another one, etc. Thus, the degree of confidence that an element $a$ is possible can be obtained by applying the "or"-operation to statements " $S$ is possible" corresponding to different sets $S$ containing the element $a$.

For each such set $S$, the degree of confidence that this set $S$ is possible is equal to $\mu(S)$. Out of all possible "or"operations, we need to select the only one for which the "or"ing of infinitely many values does not lead to 1 , namely, the maximum. Thus, we conclude that $\mu(a)$ is equal to the maximum of all the values $\mu(S)$ for all sets $S$ that contain the element $a$. This is exactly what we have in the formulation of Lemma 3. 
Comment 3. At first glance, it may seem that this commonsense explanation is all we need, and there is no need for a formal proof. One should be cautioned, however, that while the above commonsense explanation is potentially applicable for all possible elements $a$, the formula from Lemma 3 is not always true.

For example, if $X=[0,1]$ and $\mu(x)=x$, then $\mu(1)=1$ but for all sets $S$ containing 1 , we have $\mu(S) \leq 0.5$. Indeed, either this set $S$ contains the point 0.5 or it does not. In the first case,

$$
\begin{gathered}
\mu(S)=\min \left(\inf _{x \in S} \mu(x), \inf _{y \notin S}(1-\mu(y))\right) \leq \inf _{x \in S} \mu(x) \leq \\
\mu(0.5)=0.5 .
\end{gathered}
$$

In the second case,

$$
\begin{gathered}
\mu(S)=\min \left(\inf _{x \in S} \mu(x), \inf _{y \notin S}(1-\mu(y))\right) \leq \inf _{y \notin S}(1-\mu(y)) \leq \\
1-\mu(0.5)=1-0.5=0.5 .
\end{gathered}
$$

In both cases, $\mu(S) \leq 0.5$, thus, $\sup _{S: 1 \in S} \mu(S) \leq 0.5$ and thus,

$$
\sup _{S: 1 \in S}<\mu(1)=1
$$

Proof of Lemma 3. To proof Lemma 3, it is sufficient to prove the following two statements:

- that for every set $S$ that contains the element $a$, we have $\mu(S) \leq \mu(a)$, and

- that there exists a set $S$ that contains the element $a$ and for which $\mu(S)=\mu(a)$.

Let us prove these two statements one by one.

$1^{\circ}$. Let us first prove that when $a \in S$, then $\mu(S) \leq \mu(a)$.

Indeed, by definition of $\mu(S)$, we have

$$
\mu(S)=\min \left(\inf _{x \in S} \mu(x), \inf _{y \notin S}(1-\mu(y))\right) \leq \inf _{x \in S} \mu(x) \leq \mu(a) .
$$

$2^{\circ}$. Let us now prove that there exists a set $S$ that contains the element $a$ and for which $\mu(S)=\mu(a)$.

As such a set, we can take $S=\{x: \mu(x) \geq 0.5\} \cup\{a\}$. For this set, for elements $x \in S$ for which $\mu(x) \geq 0.5$, we have $\mu(x) \geq 0.5$. For the element $a \in S$, we have $\mu(a)<0.5$. Thus, the smallest of the values $\mu(x)$ for all $x \in S$ is the value $\mu(a): \inf _{x \in S} \mu(x)=\mu(a)$. For elements $y \notin S$, we have $\mu(y)<0.5$, thus $1-\mu(y)>0.5$ and hence,

$$
\inf _{y \notin S}(1-\mu(y)) \geq 0.5>\mu(a)=\inf _{x \in S} \mu(x) .
$$

So, we have

$$
\mu(S)=\min \left(\inf _{x \in S} \mu(x), \inf _{y \notin S}(1-\mu(y))\right)=\mu(a) .
$$

The lemma is proven.
Lemma 4. If $\mu(a)>0.5$, then

$$
\mu(a)=1-\sup _{S: a \notin S} \mu(S)
$$

Comment. So, for elements $a$ for which $\mu(a)>0.5$, we can also uniquely reconstruct the value $\mu(a)$ from the values $\mu(S)$.

Proof of Lemma 4. We want to prove that

$$
1-\mu(a)=\sup _{S: a \notin S} \mu(S)
$$

To proof this equality, it is sufficient to prove the following two statements:

- that for every set $S$ that does not contain the element $a$, we have $\mu(S) \leq 1-\mu(a)$, and

- that there exists a set $S$ that does not contain the element $a$ and for which $\mu(S)=1-\mu(a)$.

Let us prove these two statements one by one.

$1^{\circ}$. Let us first prove that when $a \notin S$, then $\mu(S) \leq 1-\mu(a)$. Indeed, by definition of $\mu(S)$, we have

$$
\begin{aligned}
\mu(S)= & \min _{x \in S}\left(\inf _{x \in S} \mu(x), \inf _{y \notin S}(1-\mu(y))\right) \leq \\
& \inf _{y \notin S}(1-\mu(y)) \leq 1-\mu(a) .
\end{aligned}
$$

$2^{\circ}$. Let us now prove that there exists a set $S$ that does not contain the element $a$ and for which $\mu(S)=1-\mu(a)$.

As such a set, we can take $S=\{x: \mu(x) \geq 0.5\}-\{a\}$. For this set, for elements $y \notin S$, we have $\mu(y)<0.5$ and thus, $1-\mu(y)>0.5$. For the element $a \notin S$, we have $\mu(a)>0.5$ and thus, $1-\mu(a)<0.5$. Thus, the smallest of the values $1-\mu(y)$ for all $y \notin S$ is the value $1-\mu(a)$ :

$$
\inf _{y \notin S}(1-\mu(y))=1-\mu(a) .
$$

For elements $x \in S$, we have $\mu(x) \geq 0.5$, thus

$$
\inf _{x \in S} \mu(x) \geq 0.5>1-\mu(a)=\inf _{y \notin S}(1-\mu(y)) .
$$

So, we have

$$
\mu(S)=\min \left(\inf _{x \in S} \mu(x), \inf _{y \notin S}(1-\mu(y))\right)=1-\mu(a) .
$$

The lemma is proven, and so is the proposition. 


\section{AuXiliary Result: Which CRisp Set Is the Most} PROBABLE?

Discussion. In principle, we can have many different crisp sets $S$ with different degrees $\mu(S)$. A natural question is: which crisp sets $S$ are the most probable ones (i.e., the ones for which the degree $\mu(S)$ is the largest possible)?

The answer is provided by the following result.

Proposition 2. For every membership function $\mu(x)$, and for every crisp set $S$, the following two conditions are equivalent to each other:

- the set $S$ has the largest possible value $\mu(S)$, and

- the set $S$ contains all the elements a with $\mu(a)>0.5$ and does not contain any elements a with $\mu(a)<0.5$.

Comment 1. As far as elements $a$ with $\mu(a)=0.5$ are concerned, it does not matter whether we include them or not, the value $\mu(S)$ will not change.

Comment 2. This result is in good accordance with common sense. Indeed, the inequality $\mu(a)>0.5$ is equivalent to $\mu(a)>1-\mu(a)$, and the inequality $\mu(a)<0.5$ is equivalent to $\mu(a)<1-\mu(a)$. So:

- if our degree of confidence $\mu(a)$ that the element $a$ is in the desired (unknown) set $U$ is greater than the degree of confidence $1-\mu(a)$ that $a$ is not in $U$, the we add this element $a$ to the set;

- if our degree of confidence $1-\mu(a)$ that the element $a$ is not in the desired (unknown) set $U$ is greater than the degree of confidence $\mu(a)$ that $a$ is in $U$, the we do not add this element $a$ to the set;

- if the both degrees of confidence $\mu(a)$ and $1-\mu(a)$ are equal, then we can add or not add the element $a$ to the set.

\section{Proof of Proposition 2.}

$1^{\circ}$. Let us first prove that if the value $\mu(S)$ is the largest possible, then:

- the set $S$ must contain all the elements $a$ with $\mu(a)>0.5$, and

- the set $S$ cannot contain any element $a$ with $\mu(a)<0.5$. We will prove both statements by contradiction.

$1.1^{\circ}$. Let us prove, by contradiction, that if the value $\mu(S)$ is the largest possible, then the set $S$ must contain all the elements $a$ with $\mu(a)>0.5$.

Indeed, if we take $S_{0}=\{x: \mu(x) \geq 0.5\}$, then for all $x \in A$, we have $\mu(x) \geq 0.5$, thus, $\inf _{x \in S_{0}} \mu(x) \geq 0.5$.

For all $y \notin S_{0}$, we have $\mu(y)<0.5$ and thus,

$$
1-\mu(y)>0.5 \text {. }
$$

Thus,

$$
\inf _{y \notin S_{0}}(1-\mu(y)) \geq 0.5 \text {. }
$$

Hence,

$$
\mu\left(S_{0}\right)=\min \left(\inf _{x \in S_{0}} \mu(x), \inf _{y \notin S_{0}}(1-\mu(y))\right) \geq 0.5 .
$$

So, if a crisp set $S$ has the largest possible value $\mu(S)$, we must have $\mu(S) \geq \mu\left(S_{0}\right)$ and thus, $\mu(S) \geq 0.5$.

Let us now assume that this set $S$ does not contain an element $a$ with $\mu(a)>0.5$. For this element $a \notin S$, we have $1-\mu(a)<0.5$, thus

$$
\inf _{y \notin S}(1-\mu(y)) \leq 1-\mu(a)<0.5,
$$

hence

$$
\mu(S)=\min \left(\inf _{x \in S} \mu(x), \inf _{y \notin S}(1-\mu(y))\right)<0.5,
$$

which contradicts to the proven inequality $\mu(S) \geq 0.5$.

This contradiction proves that our assumption is wrong, and thus, the optimal set $S$ includes all elements $a$ with

$$
\mu(a)>0.5 \text {. }
$$

$1.2^{\circ}$. Let us now prove, by contradiction, that if the value $\mu(S)$ is the largest possible, then the set $S$ cannot contain any element $a$ with $\mu(a)<0.5$.

Indeed, in Part 1.1 of this proof, we have already shown that if a crisp set $S$ has the largest possible value $\mu(S)$, then we have $\mu(S) \geq 0.5$.

Let us now assume that this set $S$ contains an element $a$ with $\mu(a)<0.5$. For this element $a \in S$, we have $\mu(a)<0.5$, thus $\inf _{x \in S} \mu(x) \leq \mu(a)<0.5$, hence

$$
\mu(S)=\min \left(\inf _{x \in S} \mu(x), \inf _{y \notin S}(1-\mu(y))\right)<0.5,
$$

which contradicts to the proven inequality $\mu(S) \geq 0.5$.

This contradiction proves that our assumption is wrong, and thus, the optimal set $S$ cannot include any elements $a$ with

$$
\mu(a)<0.5 \text {. }
$$

$2^{\circ}$. To complete the proof, let us show that any set $S$ that contains all the elements $a$ with $\mu(a)>0.5$ and does not contain any element $a$ with $\mu(a)<0.5$ has the largest possible value $\mu(S)$.

To prove this statement, we will consider two possible cases:

- the case when there are no elements $x$ with $\mu(x)=0.5$, and

- the case when there are elements $x$ with $\mu(x)=0.5$.

$2.1^{\circ}$. In the first case, there is only one set that satisfies the above property: the set $S_{0}=\{x: \mu(x)>0.5\}$. We have already shown that for this set $S_{0}$, we have $\mu\left(S_{0}\right) \geq 0.5$, and that for all other sets $S$, we have $\mu(S)<0.5$.

Thus, the only set $S_{0}$ that satisfies the above properties indeed has the largest possible value $\mu(S)$.

$2.2^{\circ}$. Let us now consider the second case, when there is an element $x_{0}$ for which $\mu\left(x_{0}\right)=0.5$.

$2.2 .1^{\circ}$. Let us first prove that in this case, $\mu(S) \leq 0.5$ for all crisp sets. 
Indeed, if the set $S$ contains the element $x_{0}$, then we have

$$
\inf _{x \in S} \mu(x) \leq \mu\left(x_{0}\right)=0.5
$$

and thus,

$$
\mu(S)=\min \left(\inf _{x \in S} \mu(x), \inf _{y \notin S}(1-\mu(y))\right) \leq 0.5 .
$$

If the set $S$ does not contain the element $x_{0}$, then we have

$$
\inf _{y \notin S}(1-\mu(y)) \leq 1-\mu\left(x_{0}\right)=0.5
$$

and thus, also

$$
\mu(S)=\min \left(\inf _{x \in S} \mu(x), \inf _{y \notin S}(1-\mu(y))\right) \leq 0.5 .
$$

We have shown that for the set $S_{0}=\{x: \mu(x)>0.5\}$, we have $\mu\left(S_{0}\right)=0.5$. Since for all sets $S$, we have $\mu(S) \leq 0.5$, this means that 0.5 is the largest possible value of $\mu(S)$. So, the set $S_{0}$ is indeed optimal.

$2.2 .2^{\circ}$. Let us now show that any set $S$ that contains all elements $a$ with $\mu(a)>0.5$ and does not contain any elements $a$ with $\mu(a)<0.5$ is optimal, i.e., has $\mu(S)=0.5$.

Indeed, for all elements $x \in S$, we have $\mu(x) \geq 0.5$, thus $\inf _{x \in S} \mu(x) \geq 0.5$. Similarly, for all elements $y \notin S$, we have $\mu(y) \leq 0.5$, thus $1-\mu(y) \geq 0.5$ and $\inf _{y \notin S}(1-\mu(y)) \geq 0.5$. Hence,

$$
\mu(S)=\min \left(\inf _{x \in S} \mu(x), \inf _{y \notin S}(1-\mu(y))\right) \geq 0.5 .
$$

On the other hand, we have proven that $\mu(S) \leq 0.5$ for all sets $S$. Thus, we conclude that $\mu(S)=0.5$, i.e., that the set $S$ is indeed optima.

The proposition is proven.

\section{Remaining Questions: CAn the Above UNIQUENESS RESUlT BE EXTENDED TO THE InTERVAL-VALUED FuZzy CASE? General TyPE-2?}

Need for interval-valued membership functions. An expert is often unable to describe his/her degree of confidence by a single number. In such situations, a reasonable idea is to allow an interval of possible values of degree of confidence.

Such interval-valued membership functions $\mu(x)=$ $[\mu(x), \bar{\mu}(x)]$ have been indeed successfully used in many applications; see, e.g., [2], [3], [4].

In the interval case, it is natural to define $1-[\underline{a}, \bar{a}]$ as the set of all the values $1-a$ when $a \in[\underline{a}, \bar{a}]$. The result is the interval

$$
1-[\underline{a}, \bar{a}]=[1-\bar{a}, 1-\underline{a}] .
$$

Similarly, it is natural to define $\min ([\underline{a}, \bar{a}],[\underline{b}, \bar{b}])$ as the set of all the values $\min (a, b)$ when $a \in[\underline{a}, \bar{a}]$ and $b \in[\underline{b}, \bar{b}]$. The result is the interval

$$
\min ([\underline{a}, \bar{a}],[\underline{b}, \bar{b}])=[\min (\underline{a}, \underline{b}), \min (\bar{a}, \bar{b})] .
$$

Formulation of the problem. In the interval-valued case, we can similarly define, for each crisp set $S$, the interval $\mu(S)$ as follows:

$$
\mu(S)=\min \left(\inf _{x \in S} \mu(x), \inf _{y \notin S}(1-\mu(y))\right),
$$

where $1-\mu(x)$, min, inf are now interpreted as appropriate operations with intervals.

It is then reasonable to ask a similar question: once we know the intervals $\mu(S)$ corresponding to all possible crisp sets $S$, can we uniquely reconstruct the original interval-valued membership function $\mu(x)$ ?

A similar question can be formulated when we consider type-2 fuzzy sets, when each value $\mu(x)$ is not necessarily an interval, but can be any fuzzy number.

\section{ACKNOWLEDGMENT}

This work was supported in part by the US National Science Foundation grants HRD-0734825, HRD-1242122, and DUE-0926721, and the Faculty of Economics, Chiang Mai University, Thailand.

The authors are thankful to the anonymous referees for valuable suggestions.

\section{REFERENCES}

[1] G. Klir and B. Yuan, "Fuzzy Sets and Fuzzy Logic", Prentice Hall, Upper Saddle River, New Jersey, 1995.

[2] J. M. Mendel, Uncertain Rule-Based Fuzzy Logic Systems: Introduction and New Directions, Prentice-Hall, Upper Saddle River, 2001.

[3] J. M. Mendel and D. Wu, Perceptual Computing: Aiding People in Making Subjective Judgments, IEEE Press and Wiley, New York, 2010.

[4] H. T. Nguyen, V. Kreinovich, and Q. Zuo, "Interval-valued degrees of belief: applications of interval computations to expert systems and intelligent control", International Journal of Uncertainty, Fuzziness, and Knowledge-Based Systems (IJUFKS), 1997, Vol. 5, No. 3, pp. 317-358.

[5] H. T. Nguyen and E. A. Walker, A First Course in Fuzzy Logic, Chapman and Hall/CRC, Boca Raton, Florida, 2006.

[6] L. A. Zadeh, "Fuzzy sets", Information and Control, 1965, Vol. 8, pp. $338-353$. 